TRANSACTIONS OF THE

AMERICAN MATHEMATICAL SOCIETY

Volume 350, Number 8, August 1998, Pages 3257-3268

S 0002-9947(98)01923-0

\title{
CRITERIA FOR $\bar{d}$-CONTINUITY
}

\author{
ZAQUEU COELHO AND ANTHONY N. QUAS
}

\begin{abstract}
Bernoullicity is the strongest mixing property that a measuretheoretic dynamical system can have. This is known to be intimately connected to the so-called $\bar{d}$ metric on processes, introduced by Ornstein. In this paper, we consider families of measures arising in a number of contexts and give conditions under which the measures depend $\bar{d}$-continuously on the parameters. At points where there is $\bar{d}$-continuity, it is often straightforward to establish that the measures have the Bernoulli property.
\end{abstract}

\section{INTRODUCTION}

One of the most standard examples of a measure-preserving transformation in ergodic theory is that of a Bernoulli shift. To construct these, one starts with a finite set $S$ equipped with a measure $m$ and defines the shift transformation $T$ on the space $X=S^{\mathbb{Z}}$ equipped with the measure $m^{\mathbb{Z}}$, the direct product of copies of $m$ indexed by the integers. These transformations model the simple situation which occurs in probability theory of having a sequence of independent identically distributed random variables (Bernoulli trials). For this reason, Bernoulli shifts are often referred to as the most random possible dynamical systems.

A fundamental question which often arises in ergodic theory is when a pair of measure-preserving transformations are measure-theoretically isomorphic. These questions are known to be hard, and until the mid 1970s, the results were few. It then came as a great surprise when Ornstein showed in a series of papers (collected together as [11] - see also [16]) that there is a very simple classification of Bernoulli shifts (namely, that two Bernoulli shifts are isomorphic if and only if they have the same entropy) and gave verifiable conditions for checking whether other systems are also isomorphic to Bernoulli shifts. This resulted in an explosion of work, showing that a large number of 'naturally occurring' measure-preserving systems were isomorphic to Bernoulli shifts. When this is the case, it is often said that the system has the Bernoulli property. Some of the results in this paper fall into this category.

A convenient way of establishing Bernoullicity in our context is provided by means of the $\bar{d}$ distance introduced by Ornstein. A useful description of this is offered by joinings (see Rudolph's book [14] for details). A joining of $\left(X, \nu_{1}\right)$ and $\left(X, \nu_{2}\right)$ is a shift-invariant measure on $X \times X$ with the property that $\mu(A \times X)=$ $\nu_{1}(A)$ and $\mu(X \times B)=\nu_{2}(B)$ for any measurable subsets $A$ and $B$ of $X$. Writing

Received by the editors March 7, 1996 and, in revised form, September 18, 1996.

1991 Mathematics Subject Classification. Primary 28D05, 60G10.

Key words and phrases. Bernoulli, coupling, $g$-measure.

(C)1998 American Mathematical Society 
$J\left(\nu_{1}, \nu_{2}\right)$ for the set of joinings of $\left(X, \nu_{1}\right)$ and $\left(X, \nu_{2}\right)$, the $\bar{d}$ distance is given by

$$
\bar{d}\left(\nu_{1}, \nu_{2}\right)=\inf _{\mu \in J\left(\nu_{1}, \nu_{2}\right)} \int \delta\left(x_{1}, x_{2}\right) d \mu\left(x_{1}, x_{2}\right),
$$

where $\delta(x, y)$ is 1 if the zeroth coordinates of $x$ and $y$ agree, and 0 otherwise. We will make use of this definition in the first section of this paper. The attraction of the $\bar{d}$ metric is that many ergodic properties are particularly well-behaved with respect to this metric. In particular, the set of processes which are isomorphic to Bernoulli shifts is $\bar{d}$-closed (see [11]). This will let us show that certain systems have the Bernoulli property by showing that they can be arbitrarily well approximated in the $\bar{d}$ metric by processes which have the Bernoulli property. It is important to note that Ornstein's results upon all of which this is based apply only to invertible dynamical systems. Thus, when applied to systems which are inherently one-sided, the above machinery will yield conclusions about their natural extensions. This is all that one can realistically expect in almost all cases, as the property of being one-sidedly isomorphic to a Bernoulli shift is known to be very unusual (with such nice examples as Markov chains failing to have this property - see [12]).

In the first section of the paper, we will consider the $\bar{d}$ distance between certain $g$-measures and will be able to deduce Bernoullicity results as a corollary. The notion of a $g$-measure was introduced to ergodic theory by Keane in 1974 (see [8]), but has a long history in probability theory, where it is variously called a chain with complete connections and a uniform martingale. We will take a non-standard definition of $g$-measures which is equivalent to the more usual definition in the case where the function $g$ is continuous. Let $\Sigma$ denote the full shift space $\{0, \ldots, k-1\}^{\mathbb{Z}^{+}}$. For $x \in \Sigma$ and $i \in\{0, \ldots, k-1\}$, let $i x$ denote the compound sequence defined by $(i x)_{n}=i$ if $n=0$ and $x_{n-1}$ otherwise. The function $g$ will be called a conditional distribution function if $g$ is a function from $\Sigma$ to $(0,1)$ and $\sum_{i=0}^{k-1} g(i x)=1$ for all $x$ in $\Sigma$. These functions are also referred to as $g$-functions. We will only consider those $g$ which are continuous here. The cylinder of those points which agree with $x$ for the first $n+1$ places (that is, the set $\left\{y: y_{i}=x_{i}, \forall i \leqslant n\right\}$ ) will be denoted by $[x]^{n}$. The measure $\mu$ is a $g$-measure if

$$
\lim _{n \rightarrow \infty} \frac{\mu\left([i x]^{n+1}\right)}{\mu\left([x]^{n}\right)}=g(i x)
$$

for all $x \in \Sigma$.

For continuous conditional distribution functions $g$, it is easy to show that there is at least one $g$-measure. In the case where $g$ is Hölder continuous, the $g$-measure is known to be unique and to have a Bernoulli natural extension. This statement also holds if the variations of $g$ are summable; here

$$
\operatorname{var}_{n} g=\sup _{\left\{x, y: x_{i}=y_{i}, \forall i \leqslant n\right\}}|g(x)-g(y)|
$$

(see [17] for details of the above statements). One of the weakest conditions known which guarantees uniqueness of $g$-measures was provided by Berbee (see [1]). Berbee also proves Bernoullicity. Our conditions are very similar, but slightly weaker in some cases and slightly stronger in others. A very minor modification shows that our results remain valid under Berbee's condition. It was for a long time an open question whether or not for every continuous conditional distribution function $g$, 
there is a unique $g$-measure, but this was settled recently by Bramson and Kalikow (see [3]), who constructed an example of a continuous conditional distribution function having two distinct $g$-measures.

The interpretation which we will use for $g$-measures is that $g(i x)$ is a Markov transition probability, giving the probability of moving from $x$ to $i x$. A $g$-measure is then an invariant measure for this Markov process. For details of this interpretation, the reader is referred to [13]. A closely related description is that a $g$-measure is a stationary distribution for a sequence $\left(X_{n}\right)_{n \in \mathbb{Z}}$ of random variables taking values in $S$ satisfying

$$
\mathbb{P}\left(X_{n}=i \mid X_{n-1}=x_{1}, X_{n-2}=x_{2}, \ldots\right)=g(i x) .
$$

The second section of the paper gives a condition for uniqueness of equilibrium states. For a continuous function $\phi$ defined on a two-sided shift space $X$, an equilibrium state for $\phi$ is an invariant measure maximizing the quantity $\int \phi d \mu+h_{\mu}(T)$, where $T$ is the shift map. By Walters $([17])$, it is known that if $\phi$ is a one-sided function (that is, one dependent only on those $x_{i}$ with $i \geqslant 0$ ) with the property that $\sum_{n} \operatorname{var}_{n} \phi<\infty$, then there is a unique equilibrium state for $\phi$ whose natural extension is Bernoulli. Extending the definition of variation to functions which are two-sided by defining $\operatorname{var}_{n} \phi=\sup \left\{|\phi(x)-\phi(y)|: x_{i}=y_{i}, \forall|i| \leqslant n\right\}$ and using the technique of Bowen ([2]) by which, to each two-sided function with summable variation, one associates a one-sided function, one may check that if the two-sided function $\phi$ satisfies $\sum n \operatorname{var}_{n} \phi<\infty$, then $\phi$ has a unique equilibrium state which is Bernoulli. Moreover, if $\phi$ satisfies $\sum \operatorname{var}_{n} \phi<\infty$, then $\phi$ has a unique equilibrium state and this equilibrium state is known to have the K property. This suggests the conjecture that in fact the condition $\sum \operatorname{var}_{n} \phi<\infty$ is sufficient to ensure Bernoullicity of the unique equilibrium state. This turns out to be the case, as we will show in Theorem 2.

In the final section, we will consider Gibbs measures. These were introduced by Dobrushin, Lanford and Ruelle (and are thus sometimes called DLR measures). For a detailed general reference, the reader is referred to [6].

We will consider exclusively Gibbs measures where the index set is the onedimensional lattice $\mathbb{Z}$. The state space at each site is a fixed set $S$, and so the configuration space or state space for the whole system is $S^{\mathbb{Z}}$. The Gibbs states are defined approximately by assigning energy $H(x)$ to configurations and defining a measure which is in some sense proportional to $\exp (-H(x))$ for the state $x$.

To be more precise, the energy of a configuration is defined by specifying the contribution to the total energy of each finite part of the configuration. Given $\Lambda$, a finite subset of $\mathbb{Z}, \Phi_{\Lambda}$ is a function of $S^{\mathbb{Z}}$ which is dependent only on $\left\{x_{i}: i \in \Lambda\right\}$. In a given state $x$, the contribution to the energy due to the interaction of $x_{i}$ with itself and the other sites is given by $\sum_{i \in \Lambda} \Phi_{\Lambda}(x)$. This series is required to be summable, so the requirement that $\sum_{i \in \Lambda}\left\|\Phi_{\Lambda}\right\|_{\infty}<\infty$ is imposed. From this it follows that given any finite set $\Lambda$, the sum $\sum_{\left\{\Lambda^{\prime}: \Lambda^{\prime} \cap \Lambda \neq \emptyset\right\}}\left\|\Phi_{\Lambda^{\prime}}\right\|_{\infty}<\infty$. The collection $\left(\Phi_{\Lambda}\right)$ is called a Gibbs interaction potential. The potential is called translation-invariant if it satisfies $\Phi_{\Lambda}(x)=\Phi_{\Lambda-1}(T x)$ where $\Lambda-1$ denotes the set $\{i-1: i \in \Lambda\}$ and $T$ denotes the left-shift map. We will consider only translation-invariant interaction potentials in what follows.

A Gibbs state is then defined by specifying the conditional probabilities of the various configurations on $S^{\Lambda}$ conditional on the external configuration: 


$$
\mathbb{P}\left(x_{\Lambda} \mid x_{\Lambda^{c}}\right)=\frac{\exp \left(-H_{\Lambda}\left(x_{\Lambda} x_{\Lambda}^{c}\right)\right)}{\sum_{y_{\Lambda}} \exp \left(-H_{\Lambda}\left(y_{\Lambda} x_{\Lambda}^{c}\right)\right)},
$$

where

$$
H_{\Lambda}(x)=\sum_{\left\{\Lambda^{\prime}: \Lambda^{\prime} \cap \Lambda \neq \emptyset\right\}} \Phi_{\Lambda}(x) .
$$

The question of whether, for a given interaction potential, there exists a Gibbs state and whether it is unique is a classical problem of statistical mechanics. It can be shown that in all the cases which we consider, there is at least one Gibbs state. Dobrushin gave a condition which implies uniqueness (see [4]). A second condition which is known to imply uniqueness is that the 'interaction energy of two half-lines' is finite, that is,

$$
\sum_{\{\Lambda: \Lambda \cap(-\infty, 0) \neq \emptyset, \Lambda \cap[0, \infty) \neq \emptyset\}} \operatorname{var}\left(\Phi_{\Lambda}\right)<\infty,
$$

where $\operatorname{var}\left(\Phi_{\Lambda}\right)=\max \left(\Phi_{\Lambda}\right)-\min \left(\Phi_{\Lambda}\right)$. For a reference, see [6]. In Theorem 4, we show that this condition is sufficient to imply Bernoullicity of the unique Gibbs state.

Previous results giving conditions for Bernoullicity were given by Gallavotti (see [5]) and Ledrappier (see [9]). They showed that if the interaction potential satisfies $\sum_{\Lambda \ni 0}\left\|\Phi_{\Lambda}\right\|_{\infty} \operatorname{diam}(\Lambda)<\infty$, then there is a unique Gibbs state which is Bernoulli. The result which we prove below is an extension of their work.

Note that by Ruelle's work (see [15]), there is a close connection between Gibbs states and equilibrium states. This and a result of Walters on equilibrium states are the main ingredients of the proof in the final section.

Note that throughout the paper, the results are proven in the case of full shifts on a symbol set $S$. This is primarily for simplicity of exposition, and the results remain true in the case of subshifts of finite type. In the second section, it has been pointed out to us that the part of the proof of Theorem 2 which shows that a potential with 2-sided summable variation is cohomologous to a 1-sided potential with summable variation remains valid in the case of a general subshift.

\section{1. $\bar{d}$ Distances AND $g$-MEASURES}

Let $\Sigma$ be $\{0, \ldots, k-1\}^{\mathbb{Z}^{+}}$, the one-sided full shift space on $k$ symbols.

Theorem 1. Suppose $g$ is a continuous conditional distribution function on $\Sigma$ with the property that

$$
\sum_{n=r}^{\infty} \prod_{i=r}^{n}\left(1-a_{i}\right)=\infty
$$

for some $r \geqslant 1$, where $a_{i}=\frac{k}{2} \operatorname{var}_{i}(g)$, and let $\nu_{g}$ be a g-measure. Let $h_{n}$ be $a$ sequence of continuous conditional distribution functions such that $\left\|g-h_{n}\right\|_{\infty} \rightarrow 0$ as $n \rightarrow \infty$. Then if for each $n, \nu_{h_{n}}$ is an $h_{n}$-measure, we have $\bar{d}\left(\nu_{g}, \nu_{h_{n}}\right) \rightarrow 0$ as $n \rightarrow \infty$.

Proof. Let $\nu_{g}$ be a $g$-measure, let $\|h-g\|_{\infty}=\delta$ and let $\nu_{h}$ be an $h$-measure. We will then produce a joining of $\nu_{g}$ and $\nu_{h}$ to estimate the $\bar{d}$ distance between them. 
We will consider two sequences of random variables $\left(X_{n}\right)_{n \in \mathbb{Z}}$ and $\left(Y_{n}\right)_{n \in \mathbb{Z}}$ taking values in $\{0, \ldots, k-1\}$. We will write $X_{n-}$ to denote the element $\left(X_{n-1}, X_{n-2}, \ldots\right)$ of $\Sigma$, and let $Y_{n-}$ be defined similarly. The sequences $X_{0-}$ and $Y_{0-}$ will have a specified joint distribution whose marginals are $\nu_{g}$ and $\nu_{h}$ respectively. The distribution of the $\left(X_{n}\right)$ and $\left(Y_{n}\right)$ for $n \geqslant 0$ is specified by means of a joint conditional distribution function which is constructed as follows.

Let $F:\{0, \ldots, k-1\} \times \Sigma \times \Sigma \rightarrow(0,1)$ be defined by $F(i, x, y)=\min (g(i x), h(i y))$. Define $\Delta: \Sigma \times \Sigma \rightarrow[0,1)$ by $\Delta(x, y)=1-\sum_{i} F(i, x, y)$ and note that $\Delta$ is bounded above by a constant $\alpha$ which is strictly smaller than 1 . (We can show that $\Delta$ is bounded above by $1-\inf _{x} g(x)$.) We then define a transition probability by

$$
\mathbb{P}\left(X_{n}=i, Y_{n}=i \mid X_{n-}=x, Y_{n-}=y\right)=F(i, x, y)
$$

and

$\mathbb{P}\left(X_{n}=i, Y_{n}=j \mid X_{n-}=x, Y_{n-}=y\right)=\frac{(g(i x)-F(i, x, y))^{+}(h(j y)-F(j, x, y))^{+}}{\Delta(x, y)}$

when $i \neq j$ and $a^{+}$denotes $\max (a, 0)$. It may then be checked that the marginal transition probabilities of the two processes are correct (i.e. that the conditional distribution function of $\left(X_{n}\right)$ is $g$ and the conditional distribution function of $\left(Y_{n}\right)$ is $h)$. This transition probability defines a mapping of the distributions of $\left(X_{i}, Y_{i}\right)_{i<n}$ to the distributions of $\left(X_{i}, Y_{i}\right)_{i<n+1}$. Identifying each of these spaces with the space $\mathcal{P}(\Sigma \times \Sigma)$ (where $\mathcal{P}(X)$ denotes the space of probability measures on $X$ ), the transition probability gives rise to a continuous affine map of $\mathcal{P}(\Sigma \times \Sigma)$. One can verify that this evolution leaves invariant the set of measures whose marginals on the first and second coordinates are respectively $\nu_{g}$ and $\nu_{h}$. Using the SchauderTychonoff theorem, it follows that there exists a joining $\mu$ of the measures $\nu_{g}$ and $\nu_{h}$ which is shift-invariant and has the property that the transition probabilities are given by the above formulae.

We proceed to get an upper estimate for $\bar{d}\left(\nu_{g}, \nu_{h}\right)$ by estimating the quantity $\int \delta(x, y) d \mu(x, y)$. To do this, define a map $\pi$ from $\Sigma \times \Sigma$ to $\mathbb{Z}^{+}$sending the pair $(x, y)$ to $\sup \left\{n \geqslant 0: x_{i}=y_{i}, \forall i<n\right\}$ and note that $\delta(x, y)=1$ if and only if $\pi(x, y)=0$. We then get a process $\left(Z_{n}\right)_{n \in \mathbb{Z}}$ taking values in $\mathbb{Z}^{+}$by defining $Z_{n}=\pi\left(X_{n-}, Y_{n-}\right)$. To estimate $\int \delta(x, y) d \mu$, we see that it is sufficient to estimate $\int \chi_{[0]} d \mu^{\prime}$, where $\mu^{\prime}$ is the induced measure on the $\mathbb{Z}^{+}$-valued process. Note also that in the $\mathbb{Z}^{+}$-valued process, the only valid transitions from $n$ are to 0 and $n+1$. We then estimate the transition probability of going from $n$ to 0 . We have that the transition probability is bounded above by $\sup _{\left\{x, y:[x]^{n-1}=[y]^{n-1}\right\}} \Delta(x, y)$. But we have $\Delta(x, y) \leqslant \alpha$, and also

$$
\begin{aligned}
\Delta(x, y) & =1-\sum_{i} \min (g(i x), h(i y)) \\
& =\sum_{i}(g(i x)-h(i y))^{+} \\
& \leqslant \sum_{i}(g(i x)-g(i y))^{+}+(g(i y)-h(i y))^{+} \\
& \leqslant \frac{k}{2}\left(\operatorname{var}_{n+1} g+\|g-h\|_{\infty}\right) .
\end{aligned}
$$


So we let $q_{n}=\min \left(\alpha, \frac{k}{2}\left(\operatorname{var}_{n+1} g+\|g-h\|_{\infty}\right)\right)$. This is an upper bound for the transition probability of going from $n$ to 0 . This allows us to get an upper bound on the proportion of time the $\mathbb{Z}^{+}$-valued system spends in the 0 state, thus giving an estimate on the $\bar{d}$ distance from $\nu_{g}$ to $\nu_{h}$. We have seen that the proportion of time spent in the 0 state for the $\mathbb{Z}^{+}$-valued process is bounded above by the proportion of time spent in the 0 state for the Markov chain with probabilities calculated above. It is a straightforward calculation that for this Markov chain, the limiting proportion of time spent in the 0 state is given by

$$
\begin{aligned}
p_{0} & =\left(1+\sum_{n=1}^{\infty} \prod_{i=1}^{n}\left(1-q_{i}\right)\right)^{-1} \\
& =\left(1+\sum_{n=1}^{\infty} \prod_{i=1}^{n} \min \left(1-\alpha, 1-a_{i}-\frac{k}{2}\|g-h\|_{\infty}\right)\right)^{-1}
\end{aligned}
$$

We need to show that $p_{0}$ converges to 0 as $\|g-h\|_{\infty}$ converges to 0 . To show this, we first note that since the summands in the right hand side are monotonically decreasing functions of $\|g-h\|_{\infty}$, we can swap the order of taking limits to deduce that as $\|g-h\|_{\infty} \rightarrow 0$,

$$
p_{0} \rightarrow\left(1+\sum_{n=1}^{\infty} \prod_{i=1}^{n} \min \left(1-\alpha, 1-a_{i}\right)\right)^{-1} .
$$

We therefore have $\bar{d}$-continuity of the map sending $h$ to $\nu_{h}$ at $g$ provided that

$$
\sum_{n=1}^{\infty} \prod_{i=1}^{n} \min \left(1-\alpha, 1-a_{i}\right)=\infty .
$$

This is easily seen to be equivalent to (2).

Note that one can also derive the theorem under Berbee's conditions ([1]). It is then necessary to use a different bound for $\Delta(x, y)$. Namely, one needs to use the result that $\Delta(x, y) \leqslant 1-s_{n}+\|(h-g) / g\|$, where $s_{n}=\inf _{[x]^{n}=[y]^{n}} g(x) / g(y)$.

The theorem has the following corollary.

Corollary. Suppose $g$ is a continuous conditional distribution function which satisfies (2). Then $\mu_{g}$ is the unique g-measure and has a Bernoulli natural extension.

Proof. Note that it is sufficient to show that any $g$-measure has a Bernoulli natural extension. Then, since the $g$-measures form a compact convex set, the non-extreme points of which are not even ergodic, the uniqueness will follow.

Now, let $\nu_{g}$ be a $g$-measure and let $h_{n}$ be a sequence of Hölder continuous conditional distribution functions converging to $g$ uniformly. Then the $h_{n}$-measures $\nu_{h_{n}}$ have Bernoulli natural extensions and by the theorem converge to $\nu_{g}$ in the $\bar{d}$ metric. Since the Bernoulli processes are closed in the $\bar{d}$ metric, it follows that $\nu_{g}$ has a Bernoulli natural extension, as required.

\section{Bernoullicity of Equilibrium States}

Theorem 2. Suppose $\phi$ is a two-sided function satisfying $\sum \operatorname{var}_{n} \phi<\infty$. Then there is a continuous one-sided function $\tilde{\phi}$ with the properties

1. $\sum \operatorname{var}_{n} \tilde{\phi}<\infty$;

2. $\tilde{\phi}$ is cohomologous to $\phi$. 
It follows that the equilibrium state of $\phi$ is unique and is Bernoulli.

Proof. If $\phi$ is constant, the conclusion holds trivially, so we assume that $\phi$ is not constant. Since $\phi$ is necessarily continuous, it follows that for all sufficiently large $i$, there exist numbers $n_{i}$ such that $\operatorname{var}_{n_{i}}(\phi) \leqslant 2^{-i}$, but $\operatorname{var}_{n_{i}-1}(\phi)>2^{-i}$. Set $n_{0}$ and all undefined $n_{i}$ to be 0 . Let $n_{i_{0}}$ be the first non-zero value. Note that since $\operatorname{var}_{n}(\phi)$ is a decreasing sequence, it follows that $\sum \operatorname{var}_{n}(\phi) \geqslant \sum_{i=i_{0}}^{\infty}\left(n_{i}-n_{i-1}\right) 2^{-i}=$ $\sum_{i=0}^{\infty} 2^{-(i+1)} n_{i}$. In particular, we see that

$$
\sum_{i=0}^{\infty} n_{i} 2^{-i}<\infty
$$

Now let $\phi_{i}$ be defined by $\phi_{i}(x)=\inf _{\left\{y: y_{-n_{i}}^{n_{i}}=x_{-n_{i}}^{n_{i}}\right\}} \phi(y)$ for $i \geqslant i_{0}$ and $\phi_{i}(x)=A$ (where $A=\inf _{y} \phi(y)$ ) otherwise. Then we see that $\phi_{i}$ is a sequence of functions which converges uniformly and monotonically to $\phi$. Further, $\phi_{i}$ is dependent only on $x_{-n_{i}}, \ldots, x_{n_{i}}$. Next set $\psi_{i}(x)=\phi_{i}(x)-\phi_{i-1}(x)$. Then we have $\phi(x)=A+$ $\sum_{i \geqslant i_{0}} \psi_{i}(x)$. Further, since $\phi_{i-1}(x) \leqslant \phi_{i}(x) \leqslant \phi(x)$, we see that $\left\|\psi_{i}\right\|_{\infty} \leqslant 2^{-(i-1)}$ when $i>i_{0}$.

We then define

$$
\tilde{\phi}(x)=A+\sum_{i=i_{0}}^{\infty} \psi_{i} \circ T^{n_{i}} .
$$

This is easily seen to depend only on $\left(x_{i}\right)_{i \geqslant 0}$, so is one-sided as required. It is also the uniform limit of continuous functions, so is itself continuous. We then get a bound on the variations by noting that

1. $\operatorname{var}_{n}\left(\psi_{i} \circ T^{n_{i}}\right)=0$ for $n>2 n_{i}$;

2. $\operatorname{var}_{n}\left(\psi_{i} \circ T^{n_{i}}\right) \leqslant 2^{-(i-1)}$ for $n \leqslant 2 n_{i}$ when $i>i_{0}$.

Now summing, we have $\operatorname{var}_{2 n_{i}+1}(\tilde{\phi}) \leqslant 2^{-(i-2)}$ for $i \geqslant i_{0}$. Now since the variation is decreasing, we see that

$$
\sum_{n=0}^{\infty} \operatorname{var}_{n}(\tilde{\phi}) \leqslant\left(1+2 n_{i_{0}}\right)\left(\left\|\psi_{i_{0}}\right\|_{\infty}+2\right)+\sum_{i=i_{0}}^{\infty} 2\left(n_{i+1}-n_{i}\right) 2^{-(i-2)} .
$$

In particular, it follows from (3) that $\tilde{\phi}$ has summable variation as required. It therefore follows from Walters' theorem ([17]) that the equilibrium states for $\tilde{\phi}$ are unique and Bernoulli as required. It remains only to show that $\tilde{\phi}$ is cohomologous to $\phi$. From this, it follows that the equilibrium states for $\phi$ and $\tilde{\phi}$ are the same (see [18]) To this end, define

$$
F(x)=\sum_{i=i_{0}}^{\infty} \sum_{j=0}^{n_{i}-1} \psi_{i} \circ T^{j} .
$$

First, we note that $F$ is the uniform limit of continuous functions: to see this, note that $\left\|\psi_{i} \circ T^{j}\right\|_{\infty} \leqslant 2^{-(i-1)}$ when $i>i_{0}$, so that $\sum_{i=i_{0}}^{\infty} \sum_{j=0}^{n_{i}-1}\left\|\psi_{i} \circ T^{j}\right\|_{\infty} \leqslant$ $n_{i_{0}}\left\|\psi_{i_{0}}\right\|_{\infty}+\sum_{i=i_{0}+1}^{\infty} n_{i} 2^{-(i-1)}$. We then see that $\tilde{\phi}=\phi+F \circ T-F$. It follows that $\tilde{\phi}$ is cohomologous to $\phi$ as claimed, and the result follows.

The reader should note that the above construction can also be used to show that any two-sided potential has a continuous one-sided potential which is the limit of potentials cohomologous to the original two-sided potential. 
We now use the earlier section to find sufficient conditions for the equilibrium state to vary $\bar{d}$-continuously with the potential. Set $A=\left\{\psi: \sum_{n} n \operatorname{var}_{n} \psi<\infty\right\}$ and $A_{0}=\left\{\psi: P(\psi)=0, \sum_{n} n \operatorname{var}_{n} \psi<\infty\right\}$. Equip the spaces $A$ and $A_{0}$ with the metric $d_{A}$ arising from the norm:

$$
\|\phi\|=\|\phi\|_{\infty}+\sum_{n} n \operatorname{var}_{n} \phi .
$$

Theorem 3. The map $E: A \rightarrow M(X)$ sending a potential to its equilibrium state is continuous with respect to the metrics $d_{A}$ on $A$ and $\bar{d}$ on $M(X)$.

Proof. First note that the map $\kappa: A \rightarrow A_{0}$ defined by $\kappa(\psi)=\psi-P(\psi)$ is continuous with respect to $d$, since $P$ is Lipschitz with respect to the uniform norm. Further, $\psi$ and $\kappa(\psi)$ have the same equilibrium state, so it is sufficient to work with potentials having pressure 0 .

We fix a potential $\phi$ in $A_{0}$ and note that an argument similar to that in Theorem 2 shows that since $\sum_{n} n \operatorname{var}_{n} \phi<\infty$, then $\phi$ is cohomologous to a one-sided potential $\tilde{\phi}$ which satisfies the same condition. We then observe that, given a sequence of potentials $\phi_{n}$ which converge to $\phi$ in the metric $d_{A}$, one may choose one-sided potentials $\tilde{\phi}_{n}$ cohomologous to $\phi_{n}$ which converge to $\tilde{\phi}$ in this metric. Once again, the equilibrium states for $\phi_{n}$ coincide with those for $\tilde{\phi}_{n}$.

It is now clearly sufficient to show that the map sending a one-sided potential with pressure 0 to its equilibrium state is continuous. We now show this. Pick $M>0$ and let $B$ denote the collection of one-sided potentials $\psi$ such that the pressure $P(\psi)$ is 0 and $\sum_{n} n \operatorname{var}_{n} \psi<M$. Then pick $\tau \in B$ and consider the Ruelle-Perron-Frobenius operator $\mathcal{L}_{\tau}: C(X) \rightarrow C(X)$ defined by

$$
\mathcal{L}_{\tau}(f)(x)=\sum_{i} \exp (\tau(i x)) f(i x) .
$$

It is well known (see [17]) that such an operator has a simple eigenvalue of 1 (since the pressure was taken to be 0 ) with an eigenfunction $h_{\tau}$. Further there is a measure $\nu_{\tau}$ with the property that for any continuous function $f, \mathcal{L}_{\tau}^{n}(f) \rightarrow h_{\tau} \int f d \nu_{\tau}$ where the convergence is in the supremum norm. Another property of this operator which we will need is that it is power bounded: there exists a $K$ such that $\left\|\mathcal{L}^{n}\right\|_{\infty} \leqslant K$ for all $n$.

For normalization, we have assumed that $\int h_{\tau} d \nu_{\tau}=1$. The equilibrium state is given by $d \mu_{\tau}=h_{\tau} d \nu_{\tau}$. This is a $g$-measure with continuous conditional distribution function $g_{\tau}=\exp (\tau) h_{\tau} / h_{\tau} \circ T$. By the proof of Bowen (see [2]), we see that the function $h_{\tau}$ has summable variation, and in particular, we see that $g_{\tau}$ satisfies (2). Applying a similar argument to the above, we have that the equilibrium state for $\psi \in B$ is a $g$-measure with continuous conditional distribution function $g_{\psi}$, defined by $g_{\psi}=\exp (\psi) h_{\psi} / h_{\psi} \circ T$ (where we normalize $h_{\psi}$ by assuming $\int h_{\psi} d \mu_{\tau}=1$ ). To check $\bar{d}$-closeness of $\mu_{\tau}$ and $\mu_{\phi}$, it is therefore sufficient, by Theorem 1 , to show that $h_{\psi}$ depends continuously in the supremum norm on $\psi$.

To this end, note that $\psi \in B$ implies (by the proof of Bowen) that $h_{\psi}$ satisfies $h_{\psi}(x) / h_{\psi}(y) \leqslant \exp \left(\sum_{n+1}^{\infty} \operatorname{var}_{i}(\psi)\right)$ when $x$ and $y$ coincide in the first $n$ coordinates. From this, we deduce that

$$
\left|h_{\psi}(x)-h_{\psi}(y)\right| \leqslant\left\|h_{\psi}\right\|_{\infty}\left|\exp \left(\sum_{n+1}^{\infty} \operatorname{var}_{i} \psi\right)-1\right|
$$


which may in turn be bounded above (using the mean value theorem and the fact that $\left.\left\|h_{\psi}\right\|_{\infty} \leqslant \exp M\right)$ by $\exp (2 M) \sum_{n+1}^{\infty} \operatorname{var}_{i}(\psi)$. This shows that $\operatorname{var}_{n}\left(h_{\psi}\right) \leqslant$ $\exp (2 M) \sum_{n+1}^{\infty} \operatorname{var}_{i}(\psi)$. Summing, we have

$$
\begin{aligned}
\sum_{n=0}^{\infty} \operatorname{var}_{n}(h) & \leqslant \exp (2 M) \sum_{n=0}^{\infty} \sum_{i=n+1}^{\infty} \operatorname{var}_{i}(\psi) \\
& =\exp (2 M) \sum_{n=0}^{\infty} n \operatorname{var}_{n}(\psi) \leqslant M \exp (2 M) .
\end{aligned}
$$

In particular, the $h_{\psi}$ for $\psi$ in $B$ form a relatively compact set by the Arzelà-Ascoli theorem. Let $C$ denote the closure (with respect to the supremum norm) of the collection of $h_{\psi}$ for $\psi \in B$. Then $C$ is compact, and for each $\epsilon>0$ and $f \in C$, there exists an $N>0$ such that $\left\|\mathcal{L}_{\tau}^{N} f-h_{\tau}\right\|_{\infty}<\epsilon / K$. There then exists an open neighbourhood $U$ of $f$ in $C$ such that for each $g \in U,\left\|\mathcal{L}_{\tau}^{N} g-h_{\tau}\right\|_{\infty}<\epsilon / K$. It then follows from the power-boundedness of $\mathcal{L}_{\tau}$ that $\left\|\mathcal{L}_{\tau}^{n} g-h_{\tau}\right\|_{\infty}<\epsilon$ for all $n>N$. Now by compactness, we see that there is an $N$ such that for each $f \in C$, $\left\|\mathcal{L}_{\tau}^{N} f-h_{\tau}\right\|_{\infty}<\epsilon$

We then have

$$
\begin{aligned}
\left\|h_{\tau}-h_{\psi}\right\|_{\infty} & \leqslant\left\|h_{\tau}-\mathcal{L}_{\tau}^{N} h_{\psi}\right\|_{\infty}+\left\|\mathcal{L}_{\tau}^{N} h_{\psi}-\mathcal{L}_{\psi}^{N} h_{\psi}\right\|_{\infty} \\
& \leqslant \epsilon+\left\|\mathcal{L}_{\tau}^{N} h_{\psi}-\mathcal{L}_{\psi}^{N} h_{\psi}\right\|_{\infty} .
\end{aligned}
$$

Since the right term converges to 0 as $\psi$ tends to $\tau$, the desired conclusion follows and the theorem is proved.

\section{Bernoullicity of Gibbs states}

In this section, we appeal once more to the result of Walters about summability of variations implying Bernoullicity, this time in the context of Gibbs states.

This is satisfactory in that it is an extension of the results of Gallavotti ([5]) and Ledrappier ([9]). It provides another example of the situation where all general conditions known to imply uniqueness of a measure in a certain class also imply its Bernoullicity. The result may be stated as follows:

Theorem 4. Suppose $\left(\Phi_{\Lambda}\right)_{\Lambda \in \mathcal{F}}$ is a translation-invariant interaction potential with the property that

$$
\sum_{\{\Lambda: \min \Lambda=0\}} \operatorname{diam}(\Lambda) \operatorname{var}\left(\Phi_{\Lambda}\right)<\infty .
$$

Then there is a unique equilibrium state for $\left(\Phi_{\Lambda}\right)$, and this equilibrium state has the Bernoulli property.

Note that the uniqueness referred to above is demonstrated in [6]. The earlier results of Gallavotti and Ledrappier proved Bernoullicity under stronger conditions which are equivalent to

$$
\sum_{\{\Lambda: \min \Lambda=0\}}|\Lambda| \operatorname{diam}(\Lambda) \operatorname{var}\left(\Phi_{\Lambda}\right)<\infty .
$$

Proof. We will use the work of Ruelle to relate the question to one of equilibrium states which will then have a very simple solution. 
Suppose the interaction potential $\left(\Phi_{\Lambda}\right)$ has the property (4). We construct an equilibrium potential $\phi$, which we also write as $A\left(\Phi_{\Lambda}\right)$, from $\left(\Phi_{\Lambda}\right)$ :

$$
\phi(x)=\sum_{\min \Lambda=0} \Phi_{\Lambda}(x)
$$

Note that $A$ is a linear map from the space of interaction potentials to the space of equilibrium potentials. We now demonstrate that $\phi$ has summable variations. First, we note that

$$
\operatorname{var}_{n} \phi \leqslant \sum_{\min \Lambda=0, \max \Lambda \geqslant n} \operatorname{var}\left(\Phi_{\Lambda}\right)
$$

Summing, we see that

$$
\begin{aligned}
\sum_{n=0}^{\infty} \operatorname{var}_{n} \phi & \leqslant \sum_{n=0}^{\infty} \sum_{\min } \sum_{\Lambda=0, \max \Lambda \geqslant n} \operatorname{var}\left(\Phi_{\Lambda}\right) \\
& =\sum_{n=0}^{\infty} \sum_{\min \Lambda=0, \max \Lambda=n}(n+1) \operatorname{var}\left(\Phi_{\Lambda}\right) \\
& =\sum_{\min \Lambda=0}(1+\operatorname{diam}(\Lambda)) \operatorname{var}\left(\Phi_{\Lambda}\right)<\infty
\end{aligned}
$$

It follows that $\phi$ has a unique equilibrium state which has the Bernoulli property ([17]), but using [6] and [15], we see that the unique Gibbs state is the same as the equilibrium state for $\phi$, completing the proof.

Finally, we remark that it is possible to get a result about $\bar{d}$ continuity of Gibbs states with respect to variation of the Gibbs potential in a suitable norm from the analagous result about equilibrium states:

Define a norm on the Gibbs potentials by

$$
\left\|\left(\Phi_{\Lambda}\right)\right\|=\sum_{\min \Lambda=0}\left(\left\|\Phi_{\Lambda}\right\|_{\infty}+(\operatorname{diam}(\Lambda)+1)^{2} \operatorname{var} \Phi_{\Lambda}\right) .
$$

We then claim that the map $A$ sending Gibbs potentials to equilibrium potentials has the property that it sends potentials with $\left\|\left(\Phi_{\Lambda}\right)\right\|<\infty$ to equilibrium potentials $\phi$ with $\|\phi\|<\infty$.

In fact, we can say more: Pick $\left(\Phi_{\Lambda}\right)$ with the property that $\left\|\left(\Phi_{\Lambda}\right)\right\|<\infty$ and let $\phi=A\left(\left(\Phi_{\Lambda}\right)\right)$. Then we see as before that $\operatorname{var}_{n} \phi \leqslant \sum_{\{\Lambda: \min \Lambda=0, \max \Lambda \geqslant n\}} \operatorname{var}\left(\Phi_{\Lambda}\right)$, so we have

$$
\begin{aligned}
\sum n \operatorname{var}_{n} \phi & \leqslant \sum_{\min \Lambda=0} \sum_{i=0}^{\operatorname{diam}(\Lambda)} i \operatorname{var}\left(\Phi_{\Lambda}\right) \\
& \leqslant \sum_{\min \Lambda=0}(\operatorname{diam} \Lambda)^{2} \operatorname{var}\left(\Phi_{\Lambda}\right) .
\end{aligned}
$$

Similarly, we have $\|\phi\|_{\infty} \leqslant \sum_{\min \Lambda=0}\left\|\Phi_{\Lambda}\right\|_{\infty}$, which together tell us that $\|\phi\| \leqslant$ $\left\|\left(\Phi_{\Lambda}\right)\right\|$, so that the linear operator $A$ has norm at most one when restricted to the space of Gibbs potentials of finite norm.

This tells us that the one-sided equilibrium potential depends continuously on the Gibbs potential, and we can therefore apply Theorem 3 to deduce 
Theorem 5. Let $\mathcal{G}$ denote the collection of Gibbs potentials for which $\left\|\left(\Phi_{\Lambda}\right)\right\|<\infty$. Then, restricted to $\mathcal{G}$ with norm $\|\cdot\|$, the map sending a Gibbs potential to its Gibbs state is continuous with respect to $\bar{d}$.

It would be interesting to see whether Bernoullicity could be established for Gibbs states satisfying the weaker conditions introduced by Minlos and Natapov in [10], which they show are sufficient to guarantee uniqueness of the Gibbs state.

In addition, it is somewhat unsatisfactory that conditions for the $\bar{d}$-continuity of the equilibrium states and Gibbs states are stronger than those required for the Bernoullicity. It seems likely that $\bar{d}$-continuity would hold under weaker conditions than those which we have been able to find so far.

The first named author would like to thank the Royal Society for financial support and the Mathematics Institute of the University of Warwick for their hospitality during part of the time while this work was being completed.

We thank Klaus Schmidt for suggesting the problem of summability of variations implying Bernoullicity of equilibrium state, and understand that he has independently obtained a different proof.

We would like to express our thanks to Peter Walters for his careful reading of this paper, and his many helpful comments which have helped improve the results and streamline the exposition.

\section{REFERENCES}

[1] H. Berbee, Chains with infinite connections: uniqueness and Markov representation, Probab. Th. Rel. Fields 76 (1987), 243-253. MR 89c:60052

[2] R. Bowen, Equilibrium states and the ergodic theory of Anosov diffeomorphisms, Lecture Notes in Math., vol. 470, Springer-Verlag, 1975. MR 56:1364

[3] M. Bramson and S. A. Kalikow, Nonuniqueness in g-functions, Israel J. Math. 84 (1993), 153-160. MR 94h:28011

[4] R. L. Dobrushin, The problem of uniqueness of a Gibbs random field and the problem of phase transition, Funct. Anal. Appl. 2 (1968), 302-312. MR 40:3863

[5] G. Gallavotti, Ising model and Bernoulli schemes in one dimension, Commun. Math. Phys. 32 (1973), 183-190. MR 50:9270

[6] H.-O. Georgii, Gibbs measures and phase transitions, de Gruyter, Berlin, 1988. MR $89 \mathrm{k}: 82010$

[7] G. R. Grimmett and D. R. Stirzaker, Probability and random processes (2nd edition), Oxford Univ. Press, Oxford, 1992. MR 93m:60002

[8] M. Keane, Strongly mixing g-measures, Invent. Math 16 (1972), 309-324. MR 46:9295

[9] F. Ledrappier, Mesures d'équilibre sur un reseau, Commun. Math. Phys. 33 (1973), 119-128. MR 50:575

[10] R. A. Minlos and G. M. Natapov, Uniqueness of the limit Gibbs distribution in onedimensional classical systems, Theor. Math. Phys. 24 (1975), 697-703. MR 58:8981

[11] D. S. Ornstein, Ergodic theory, randomness and dynamical systems, Yale Univ. Press, New Haven, 1974. MR 56:5836

[12] W. Parry and P. Walters, Endomorphisms of a Lebesgue space, Bull. Amer. Math. Soc. 78 (1972), 272-276. MR 45:3674

[13] A. N. Quas, Non-ergodicity for $C^{1}$ expanding maps and g-measures, Ergodic Theory Dynamical Systems 16 (1996), 531-543. MR 97d:28025

[14] D. J. Rudolph, Fundamentals of measurable dynamics, Oxford Univ. Press, Oxford, 1990. MR 92e:28006

[15] D. Ruelle, Thermodynamic formalism, Cambridge University Press, Cambridge, 1978. MR 80g: 82017

[16] P. C. Shields, The theory of Bernoulli shifts, University of Chicago Press, Chicago, 1973. MR 56:584 
[17] P. Walters, Ruelle's operator theorem and g-measures, Trans. Amer. Math. Soc. 214 (1975), 375-387. MR 54:515

[18] P. Walters, An introduction to ergodic theory, Springer, New York, 1981. MR 84e:28017

Instituto de Matemática e Estatítica, Universidade de São Paulo, São Paulo, Brazil

Current address: Departamento de Matemática Aplicada, Faculdade de Ciências, Universidade do Porto, Rua das Taipas 135, P-4050 Porto, Portugal

E-mail address: zcoelho@fc.up.pt

Statistical Laboratory, Department of Pure Mathematics and Mathematical Statistics, 16 Mill Lane, Cambridge, CB2 1SB, England

Current address: Department of Mathematical Sciences, University of Memphis, Memphis, Tennessee 38152

E-mail address: quasa@msci.memphis.edu 